\title{
ON THE EXISTENCE OF SOLUTIONS OF MARTINGALE INTEGRAL EQUATIONS
}

\author{
NoRIHIKO KAZAMAKI
}

(Received Feb. 17, 1972)

1. In the present paper we shall consider the following stochastic integral equation:

$$
X_{t}=x+\int_{0}^{t} f\left(X_{u-}\right) d M_{u}+\int_{0}^{t} g\left(X_{u-}\right) d A_{u}, \quad X_{0}=x \in R
$$

where $\left(M_{t}\right)$ is a local martingale and $\left(A_{t}\right)$ is an increasing process. This is a continuation of [1] in which we assumed the square integrability of each $M_{t}$ and the continuity of the process $\left(A_{t}\right)$.

2. Let $(\Omega, F, P)$ be a complete probability space, given an increasing right continuous family $\left(F_{t}\right)$ of sub $\sigma$-fields of $F$. We assume as usual that $F_{0}$ contains all the negligible sets. In addition, suppose the family $\left(F_{t}\right)$ is quasi-left continuous; namely, for every stopping time $T$ and every sequence $\left(T_{n}\right)$ of stopping times such that $T_{n} \uparrow T$, the $\sigma$-field $F_{T}$ is generated by the field $\bigcup_{n=1}^{\infty} F_{T_{n}}$. A notation such that "let $M=\left(M_{t}, F_{t}\right)$ be martingale" means that the martingale property is relative to the family $\left(F_{t}\right)$. All martingales below are assumed to be right continuous.

By a normal change of time $C=\left(F_{t}, c_{t}\right)$ we mean a family of stopping times of the family $\left(F_{t}\right)$, finite valued, such that for a.e $\omega$ the sample function $c .(\omega)$ is strictly increasing,

$$
c_{0}(\omega)=0, c_{\infty}(\omega)=\lim _{t \rightarrow \infty} c_{t}(\omega)=\infty
$$

and continuous.

As usual, we do not distinguish two processes $X$ and $Y$ such that for a.e $\omega X .(\omega)=Y \cdot(\omega)$. This is important for the understanding of uniqueness statements below.

Definition. A right continuous process $M=\left(M_{t}, F_{t}\right)$ is a local martingale if there exists a sequence of stopping times $T_{n} \uparrow \infty$ such that for every $n$ the process $M_{t \wedge T_{n}}$ on the set $\left\{T_{n}>0\right\}$ is a uniformly integrable martingale.

We assume in this paper that $M_{0}=0$. 
3. We are now in a position to state our result.

THEOREM. Let $f$ and $g$ be real valued bounded functions such that for all $x, y \in R$

$$
\operatorname{Max}(|f(x)-f(y)|,|g(x)-g(y)|) \leqq \alpha|x-y|
$$

where $\alpha$ is some constant. Then the equation (1) has a unique solution.

The key to the proof of this theorem is the following lemma which is closed to the Gundy decomposition of martingales. Since it is proved in [2], we omit the proof.

Lemma. Let $M$ be a local martingale. Then there exist stopping times $R_{n} \uparrow \infty$ such that the process $M_{t \wedge R_{n}}$ can be written as

$$
M_{t \wedge R_{n}}=H_{t}+V_{t}, V_{t}=M_{R_{n}} I_{\left\{t \geqq R_{n}\right\}}+B_{t}^{(1)}-B_{t}^{(2)}
$$

where $\left(H_{t}\right)$ is an $L^{2}$-bounded martingale stopped at $R_{n}$ and each $\left(B_{t}^{(i)}\right), i=$ 1,2 , is a natural increasing process.

Of course, $H$ and $B^{(i)}$ depend on $R_{n}$. Note that if the family $\left(F_{t}\right)$ is quasi-left continuous, then any natural increasing process is continuous; so $B^{(i)}$ is continuous. This fact is important in the following.

Proof of Theorem. Let us keep the notations used in the lemma. As is well known, there exists a unique continuous increasing process $\widetilde{A}_{t}$ such that the process $A_{t}^{*}=A_{t}-\tilde{A}_{t}$ is a martingale. Then we can rewrite the equation (1) in the form

$$
X_{t}=x+\int_{0}^{t} f\left(X_{u-}\right) d M_{u}+\int_{0}^{t} g\left(X_{u-}\right) d A_{u}^{*}+\int_{0}^{t} g\left(X_{u-}\right) d \tilde{A}_{u} .
$$

Therefore, there is no loss of generality in assuming that the process $A$ is continuous, as we now do.

First, we shall treat the equation (1) on the stochastic interval [0, $R$, where $R$ is one of the stopping times $\left(R_{n}\right)$ in the lemma. On this interval we have

$$
X_{t}=x+\int_{0}^{t} f\left(X_{u-}\right) d H_{u}+\int_{0}^{t} f\left(X_{u-}\right) d B_{u}^{(1)}-\int_{0}^{t} f\left(X_{u-}\right) d B_{u}^{(2)}+\int_{0}^{t} g\left(X_{u-}\right) d A_{u} .
$$

As is well known, there exists a unique continuous increasing process $\langle H\rangle$ such that $H^{2}-\langle H\rangle$ is a martingale.

Define now:

$$
\lambda_{t}=t+\langle H\rangle_{t}+B_{t}^{(1)}+B_{t}^{(2)}+A_{t}, \quad \theta_{t}=\inf \left\{u: \lambda_{u}>t\right\} .
$$

Clearly $\left(\lambda_{t}\right)$ is a continuous increasing process with $P\left(\lambda_{0}=0, \lambda_{\infty}=+\infty\right)=1$. 
Then an easy computation shows that $\Theta=\left(F_{t}, \theta_{t}\right)$ and $\Lambda=\left(F_{\theta_{t}}, \lambda_{t}\right)$ are normal change of time. It is obvious that $\lambda_{R}$ is a stopping time with respect to the Family $\left(F_{\theta_{t}}\right)$ and the process $\left(t-\langle H\rangle_{\theta_{t}}-B_{\theta_{t}}^{(1)}-B_{\theta_{t}}^{(2)}-A_{\theta_{t}}\right.$, $F_{\theta_{t}}$ ) is increasing. As $\theta_{t}<R$ on the set $\left\{t<\lambda_{R}\right\}$, we get from (5)

$$
\text { (7) } \begin{aligned}
X_{\theta_{t}} & =x+\int_{0}^{t} f\left(X_{\theta_{u-}}\right) d M_{\theta_{u}}+\int_{0}^{t} g\left(X_{\theta_{u-}}\right) d A_{\theta_{u}} \\
& =x+\int_{0}^{t} f\left(X_{\theta_{u}}\right) d H_{\theta_{u}}+\int_{0}^{t} f\left(X_{\theta_{u}}\right) d B_{\theta_{u}}^{(1)}-\int_{0}^{t} f\left(X_{\theta_{u-}}\right) d B_{\theta_{u}}^{(2)}+\int_{0}^{t} g\left(X_{\theta_{u}}\right) d A_{\theta_{u}}
\end{aligned}
$$

on the stochastic interval $\left[0, \lambda_{R}\right.$ [ relative to the family $\left(F_{\theta_{t}}\right)$.

Therefore, in order to show the existence of a unique solution of the equation (1) on the interval [0, $R$ [, it suffices to consider the equation (7) in stead of (1). Namely, there is no loss of generality in assuming that the process $\left(t-\langle H\rangle_{t}-B_{t}^{(1)}-B_{t}^{(2)}-A_{t}, F_{t}\right)$ is increasing, as we now do. For simplicity, the proof is spelled out for $0 \leqq t \leqq 1$ only.

Define in succession:

$$
\begin{aligned}
X_{t}^{0} & =x \\
X_{t}^{n+1} & =x+\int_{0}^{t} f\left(X_{u_{-}}^{n}\right) d M_{u}+\int_{0}^{t} g\left(X_{u^{-}}^{n}\right) d A_{u}, \quad n=1,2, \cdots .
\end{aligned}
$$

Clearly the processes $\left(f\left(X_{t}^{n}\right)\right)$ and $\left(g\left(X_{t}^{n}\right)\right)$ are right continuous.

Put now: $c_{t}^{n}=f\left(X_{t}^{n}\right)-f\left(X_{t}^{n-1}\right), d_{t}^{n}=g\left(X_{t}^{n}\right)-g\left(X_{t}^{n-1}\right)$. For simplicity, we assume that $\alpha \leqq 1 / 4$. Then, by using the Schwarz inequality, we have

$$
\begin{aligned}
D_{n}(t) \equiv & E\left[\left(X_{t}^{n+1}-X_{t}^{n}\right)^{2} I_{\{t<R\}}\right] \\
= & E\left[\left(\int_{0}^{t} c_{u-}^{n} d M_{u}+\int_{0}^{t} c_{u-}^{n} d B_{u}^{(1)}-\int_{0}^{t} c_{u-}^{n} d B_{u}^{(2)}+\int d_{u-}^{n} d A_{u}\right)^{2} I_{\{t<R\}}\right] \\
\leqq & 4 E\left[\int_{0}^{t}\left(c_{u}^{n}\right)^{2} I_{\{u<R\}} d\langle H\rangle_{u}+B_{t}^{(1)} \int_{0}^{t}\left(c_{u}^{n}\right)^{2} I_{\{u<R\}} d B_{u}^{(1)}\right. \\
& \left.+B_{t}^{(2)} \int_{0}^{t}\left(c_{u}^{n}\right)^{2} I_{\{u<R\}} d B_{u}^{(2)}+A_{t} \int_{0}^{t}\left(d_{u}^{n}\right)^{2} I_{\{u<R\}} d A_{u}\right] \\
\leqq & (4 \alpha)^{2} \int_{0}^{t} E\left[\left(X_{u}^{n}-X_{u}^{n-1}\right)^{2} I_{\{u<R\}}\right] d u \\
\leqq & \int_{0}^{t} D_{n-1}(u) d u ; D_{0}(t) \leqq(4 K)^{2} t, \text { where } K=\operatorname{Max}\left(\|f\|_{\infty},\|g\|_{\infty}\right) .
\end{aligned}
$$

As $\sup _{0 \leqq t \leqq 1} D_{0}(t) \leqq(4 K)^{2}$, we derive the estimate

$$
D_{n}(t) \leqq(4 K)^{2} \frac{t^{n+1}}{(n+1) !} \cdot
$$

Since the process $\left(\int_{0}^{t} c_{u-}^{n} d H_{u}, F_{t}\right)$ is an $L^{2}$-bounded martingale, the extension 
of Kolmogorov's inequality to martingales shows that for any $\varepsilon>0$

$$
\begin{aligned}
P\left(\sup _{0 \leqq t \leq 1}\left|\int_{0}^{t} c_{u-}^{n} d H_{u}\right|\right. & \geqq \varepsilon) \leqq \varepsilon^{-2} E\left[\int_{0}^{t}\left(c_{u}^{n}\right)^{2} d\langle H\rangle_{u}\right] \\
& \leqq \varepsilon^{-2} E\left[\int_{0}^{t}\left(c_{u}^{n}\right)^{2} I_{\{u<R \mid} d\langle H\rangle_{u}\right] \quad\left(\because H_{t}=H_{t \wedge R}\right) \\
& \leqq \alpha^{2} \varepsilon^{-2} \int_{0}^{1} D_{n-1}(u) d u .
\end{aligned}
$$

Next, we get by using the Schwarz inequality

$$
\begin{aligned}
P\left(\sup _{0 \leqq t \leqq 1}\left|\int_{0}^{t} c_{u-}^{n} d B_{u}^{(i)}\right| \geqq \varepsilon\right) & =P\left(\sup _{0 \leqq t \leqq 1}\left(\int_{0}^{t} c_{u-}^{n} d B_{u}^{(i)}\right)^{2} \geqq \varepsilon^{2}\right) \\
& \leqq P\left(\sup _{0 \leqq t \leqq 1} B_{t}^{(i)} \int_{0}^{t}\left(c_{u-}^{n}\right)^{2} d B_{u}^{(i)} \geqq \varepsilon^{2}\right) \\
& \leqq P\left(\int_{0}^{1}\left(c_{u-}^{n}\right)^{2} I_{\{u<R} d u \geqq \varepsilon^{2}\right) \quad\left(\because B_{t}^{(i)}=B_{t \wedge R}^{(i)}\right) \\
& \leqq \alpha^{2} \varepsilon^{-2} \int_{0}^{1} D_{n-1}(u) d u .
\end{aligned}
$$

Similarly we obtain

$$
P\left(\sup _{0 \leq t \leq 1}\left|\int_{0}^{t} d_{u-}^{n} d A_{u \wedge R}\right| \geqq \varepsilon\right) \leqq \alpha^{2} \varepsilon^{-2} \int_{0}^{1} D_{n-1}(u) d u .
$$

Thus $P\left(\sup _{0 \leq t \leq 1}\left|X_{t}^{n+1}-X_{t}^{n}\right| I_{|t<R|} \geqq 4 \varepsilon\right) \leqq$ Const. $\times \varepsilon^{-2} /(n+1)$ !. Pick $\varepsilon^{-2}=$ $(n-1)$ !. Then $\varepsilon^{-2} /(n+1)$ ! is the general term of a convergent sum, and so the Borel-Cantelli lemma shows that the processes $\left(X_{t}^{n} I_{i t<R]}\right)$ converge uniformly almost surely for $0 \leqq t \leqq 1$ to some right continuous process $X^{R}=\left(X_{t}^{R}, F_{t}\right)$. Furthermore by using the extension of Kolmogorov's inequality to martingales we have

$$
\begin{aligned}
& P\left(\sup _{0 \leqq t \leq 1}\left|\int_{0}^{t} f\left(X_{u-}^{n}\right) d H_{u}-\int_{0}^{t} f\left(X_{u-}^{R}\right) d H_{u}\right| \geqq \varepsilon\right) \\
& \quad \leqq \varepsilon^{-2} E\left[\int_{0}^{1}\left\{f\left(X_{u}^{n}\right)-f\left(X_{u}^{R}\right)\right\}^{2} I_{\{u<R\}} d\langle H\rangle_{u}\right] .
\end{aligned}
$$

According to the bounded convergence theorem, the right hand side of this inequality converges to 0 as $n \rightarrow \infty$. Thus the processes $\left(\int_{0}^{t} f\left(X_{u}^{n_{k}}\right) d H_{u}\right)$ converge uniformly almost surely to the process $\left(\int_{0}^{t} f\left(X_{u}^{R}\right) d H_{u}\right)$ for some subsequence $\left(n_{k}\right)$. It is not difficult to see that $\left(\int_{0}^{t} f\left(X_{u-}^{n}\right) d B_{u}^{(i)}\right)$ and $\left(\int_{0}^{t} g\left(X_{u-}^{n}\right) d A_{u \wedge R}\right)$ converge uniformly almost surely to $\left(\int_{0}^{t} f\left(X_{u_{-}}^{R}\right) d B_{u}^{(i)}\right)$ and $\left(\int_{0}^{t} g\left(X_{u-}^{R}\right) d A_{u \wedge R}\right)$ respectively. Consequently the process $X^{R}$ satisfies the following equality: 
(13)

$$
\begin{array}{r}
X_{t}^{R}=x+\int_{0}^{t} f\left(X_{u-}^{R}\right) d H_{u}+\int_{0}^{t} f\left(X_{u-}^{R}\right) d B_{u}^{(1)}-\int_{0}^{t} f\left(X_{u-}^{R}\right) d B_{u}^{(i)}+\int_{0}^{t} g\left(X_{u-}^{R}\right) d A_{u} \\
\text { on }[0, R[.
\end{array}
$$

That is, $X^{R}$ satisfies the equation (1) on the interval [0, $R$ [.

Now let $X$ and $Y$ be two solutions of the equation (1) on [0, $R$. Then we can obtain as in the proof of (9)

$$
D(t) \equiv E\left[\left(X_{t}-Y_{t}\right) I_{\{t<R\}}\right] \leqq \int_{0}^{t} D(u) d u, \quad \sup _{0 \leqq t \leqq 1} D(t) \leqq 32 K^{2},
$$

from which $D(t)=0$. This implies that $X=Y$ on $[0, R[$.

Next, for each $n$, let $X^{R_{n}}=\left(X_{t}^{R_{n}}, F_{t}\right)$ be a solution of the equation

(1) on the stochastic interval [0, $R_{n}\left[\cdot X^{R_{n+1}}\right.$ being also a solution of (1) on [0, $R_{n}$ [, we get $X^{R_{n}}=X^{R_{n+1}}$ on $\left[0, R_{n}[\right.$. This relation therefore defines a right continuous process $X$ such that

$$
X=X^{R_{n}} \text { on }\left[0, R_{n}[, n=1,2, \cdots\right.
$$

Furthermore, for each $n$,

$$
\left.E\left[\left\{\int_{0}^{t}\left(f X_{u-}^{R_{n}}\right)-f\left(X_{u-}\right)\right) d H_{u}\right\}^{2}\right]=E\left[\int_{0}^{t}\left\{f\left(X_{u-}^{R_{n}}\right)-f\left(X_{u-}\right)\right\}^{2} I_{\left\{u<R_{n}\right\}} d\langle H\rangle_{u}\right]=0,
$$

from which $\int_{0}^{t} f\left(X_{u-}\right) d H_{u}=\int_{0}^{t} f\left(X_{u}^{R_{n}}\right) d H_{u}$ on $\left[0, R_{n}[\right.$.

Obviously we have on the interval $\left[0, R_{n}\right.$ [

$$
\int_{0}^{t} f\left(X_{u-}\right) d B_{u}^{(i)}=\int_{0}^{t} f\left(X_{u-}^{R_{n}}\right) d B_{u}^{(i)} \text { and } \int_{0}^{t} g\left(X_{u-}\right) d A_{u}=\int_{0}^{t} g\left(X_{u-}^{R_{n}}\right) d A_{u} \cdot
$$

Thus, the process $X$ satisfies the equation (1) on each $\left[0, R_{n}\left[. \quad\right.\right.$ As $R_{n} \uparrow \infty$, $X$ is a solution of (1).

Finally, we are going to show its uniqueness. If $X$ and $Y$ are two solutions of (1), then these processes satisfy the equation (1) on each interval [0, $R_{n}\left[\right.$. Therefore $X=Y$ on $\left[0, R_{n}[\right.$ for each $n$. Hence $X=Y$. This completes the proof.

4. In the following, instead of the quasi-left continuity of the family $\left(F_{t}\right)$, we assume that the local martingale $M$ and the increasing process $A$ are continuous.

Proposition. Let $\rho$ and $\kappa$ be positive increasing function defined on $(0, \infty)$. Suppose that

$$
\begin{aligned}
& \int_{0+} \rho^{-2}(u) d u=+\infty, \quad \int_{0+} \kappa^{-1}(u) d u=+\infty \\
& \quad|f(x)-f(y)| \leqq \rho(|x-y|), \quad|g(x)-g(y)| \leqq \kappa(|x-y|), \quad \forall x, y \in R
\end{aligned}
$$


and $\kappa$ is concave.

Then the uniqueness holds for the equation (1).

By using a normal change of time, this proposition can be proved in the same way as Theorem 1 of [3].

\section{REFERENCES}

[1] N. KAZAMAKI, Note on a stochastic integral equation, Univesité de Strasbourg, Lecture Notes in Mathematics, Springer, Heidelberg. (to appear)

[2] C. Dolf́ans-DAde AND P. A. MEYer, Intégrales stochastiques par rapport aux martingales locales, Université de Strasbourg, Lecture Notes in Mathematics, vol. 124, Springer, Heidelberg (1970), 77-107.

[3] T. Yamada and S. Watanabe, On the uniqueness of solutions of stochastic differential equations, J. Math. Kyoto Univ. II-1 (1971), 155-167.

Mathematical Institute

TOHOKU UNIVERSITY

SENDAI, JAPAN 